\title{
Time and space matter: how urban transitions create inequality
}

\author{
François Gusdorf ${ }^{\mathrm{a}, \mathrm{b}}$, Stéphane Hallegatte ${ }^{\mathrm{a}, \mathrm{b}, *}$ Alain Lahellec ${ }^{\mathrm{c}}$ \\ ${ }^{a}$ Centre International de Recherche sur l'Environnement et le Développement, \\ Paris, France ${ }^{1}$ \\ ${ }^{\mathrm{b}}$ Ecole Nationale des Ponts-et-Chaussées, Paris, France \\ ${ }^{\mathrm{c}}$ Laboratoire de Météorologie Dynamique, Université de Jussieu, Paris, France
}

\begin{abstract}
To analyze the response of cities to urban policies or transportation shocks, describing a succession of stationary states is not enough, and urban dynamics should be taken into account. To do so, the urban economics model NEDUM is proposed. This model reproduces the evolution of a monocentric city in continous time and captures the interaction between household moves, changes in flat sizes, rent levels, and density of housing service supply. NEDUM allows, therefore, for a temporal and spatialized analysis of urban transitions. Applied to climate policies, this model suggests that the implementation of a transportation tax causes a larger welfare loss than can be inferred from traditional models. Moreover, such a tax increases significantly inequalities if its implementation is not anticipated enough. According to these results, therefore, smooth and early implementation paths of climate policies should be favored over delayed and aggressive action.
\end{abstract}

Key words: City, Housing, Transportation

\section{Introduction}

As mobility needs induce a large and increasing share of greenhouse gas emissions, it is very likely that transportation systems will have to change if our societies are to respect a carbon constraint. Urban transportation systems

* Corresponding author. Tel.: 331439473 65, Fax.: 331439473 70. CIRED, 45bis Av. de la Belle Gabrielle, F-94736 Nogent-sur-Marne, France.

Email address: hallegatte@centre-cired.fr (Stéphane Hallegatte).

1 CIRED is a EHESS-CNRS-ENPC-ENGREF laboratory.

Preprint submitted to Elsevier Science 9 August 2007 
will, therefore, have to go through significative evolutions, and ambitious urban policies are indeed a necessary step so as to achieve Kyoto-like objectives (Srinivas, 2000).

Economic evaluations of climate policies often consist in the assessment of aggregated GDP losses (e.g. Tulkens and Tulkens, 2006). The analyzis of urban policies, however, shall tackle impacts that are widely differentiated in space. The effects of an increase in oil prices on suburban households that are heavily dependant on private vehicles, for instance, should be distinguished from the effects on central city inhabitants. Cities, moreover, are slowly-evolving systems: assessing the cost of a change in transportation systems not only requires knowledge about the present and future equilibriums of the system; it also requires an analysis of the transition paths between these equilibriums, and a specific investigation of the important question of inertia (Rotmans et al., 1994) applied to urban dynamics.

This paper aims at providing a framework to assess transitions of urban systems, including its spatial distribution. Beyond its general interest, the question of differentiated effects in time and space of energy policies is particularly crucial for cities. The functioning of cities relies on long-lived infrastructures in housing and transportation sectors, that can only adapt gradually to new economic conditions (Gusdorf and Hallegatte, 2007). But infrastructures are not the only source of inertia: it also takes time to households to change their locations, to modify their consumption bundles, and housing rents are also sticky to a certain extent.

We propose here a model that is fitted to address the stylized evolutions of urban systems through time and space. This Non Equilibrium Dynamic Urban Model (NEDUM) is based on the classic urban model à la Von Thuenen (1826), adapted to cities by Alonso (1964), Mills (1967) and Muth (1969). Dynamic analysis of cities based on the Von Thuenen framework have already been proposed before, but they only consisted in a sequence of stationary equilibriums, see e.g. Anas (1978) or Capozza and Helsley (1990), and a review in Brueckner (2000). Our approach is innovative in that it allows to represent non-stationary states, taking into account inertia in households relocation, in apartments' sizes, housing service production, and stickiness in housing rents.

In addition, we introduce macroeconomic feedbacks in the model by making income endogenous: workers supply their labor force to firms that produce a composite goods, a process we represent through a neo-classical production function. A constant share of product is saved, and used for investments. Investments are either directed towards the productive or the housing sector, depending on their respective profitability. This interdependence between investment choices allows for the representation of crowding-out effects when housing needs make construction more profitable than productive investments. 
We use NEDUM to perform two sets of numerical experiments. First, we simulate the effects of a shock on transportation costs. We show that the distribution in time of the cost of such a shock is very unequally spread between the short, medium and long run. Long term effects are classical results, and we focus our analysis on transition phenomenons. With our calibration, roughly based on the characteristics of the Los Angeles agglomeration, a 50\% increase in transportation costs leads to significant negative effects on utility levels during approximately 60 years after the shock. Households living at the outskirts of the city are most impacted during the transition period. We quantify this effect with the Gini index, which is a common economic tool designed to quantify inequalities ${ }^{2}$ : in our stylized city, though we assumed that all workers earn the same income, this index stays above 0.02 during approximately 55 years after the shock, and reaches a peak value close to 0.12 . This is indeed a strong effect, justifying the need for extensive analysis of the effects of urban transitions.

Second, we assume that the city government has decided to implement a transportation tax (Collier and Loefstedt, 1997), that will represent a $50 \%$ increase in transportation costs in year 2050. Before this date, the implementation path is freely chosen by the government, and we investigate the advantages of early and smooth, vs. late, and aggressive action. Early implementation allows to cope with the inertia of several mechanisms; however, it imposes an early constraint on economic agents, which may worsen the situation compared to late implementation. We find that there exists an equity vs. efficiency trade-off, since implementing the signal-price in less that 20 years may reduce welfare costs compared to early implementation, but entails significant anti-redistributive effects. These results show that a part of the population is strongly impacted by the changes in urban systems, and could be deeply opposed to these changes. The taking into account of this mechanism may be as important for policy design as the aggregate economic costs.

The remainder of this paper is as follows: Section 2 is a brief reminder of the classic urban model à la Von Thuenen. In Section 3, we present the NEDUM model, that is exposed in details in the Appendix A and B. Section 4 investigates the effects of a shock on transportation costs, and Section 5 analyses various implementation paths of a given transport taxation level. Finally, Section 6 concludes and provides insights for future research.

2 In brief, the Gini index varies between 0 and 1 ; it is equal to 0 when there are no inequalities, and increases with inequality, to reach 1 when one individual earns the entire income of the society. 


\section{The traditional equilibrium urban model}

In this section, the general features of the classical static equilibrium framework describing urban systems are recalled. We do it briefly because it is a traditional model, which reproduces some well established stylized observations about cities (Wheaton, 1974). A more detailed description can be found for instance in Fujita (1989).

In this stylized monocentric city, housing is organized around a Central Business District (CBD). A given number $N$ of identical households inhabits the city: in each household there is one worker commuting every day to and from the CBD, earning the same income, of which they derive the consumption level $c$. Transportation costs with respect to the distance $r$ from the CBD are given, while housing rents are endogenous, and ensure that identical households reach the same utility level at the equilibrium, even though they live at different locations. Household behavior is driven by the maximization of a utility function $U(z, q)$ describing preferences for the consumption of composite goods $z$ and housing service $q$ :

$$
\max _{r, z, q} U(z, q)
$$

s.t.

$$
z+R_{H}(r) q \leq c-T(r)
$$

Equation (2) is the budget constraint of the household, and the composite goods is chosen as the numeraire. The variable $R_{H}(r)$ is the rent level per unit of housing service at distance $r$ from the CBD.

We use a production function of housing service $F(K, L)$ à la Muth (1969): this function $F$ takes capital $K$ and land $L$ as inputs, and is linear. We specify the housing service density $h(r)=H(r) / \operatorname{Land}(r)=f\left(x^{*}(r)\right)$, where $x=K / L$. The function $f$ is defined by $f(x)=F(1, x)$, and the superscript "**" denotes equilibrium values.

Generalized transportation costs are represented by the function $T(r)$, that takes into account the cost of transportation itself as well as the cost of the time spent in commuting, which otherwise could have been devoted to work.

Table 2 presents the standard nomenclature we use, while Eqs. (1) to (7) describe the basic relationships of the classical urban modeling framework.

$$
\max _{K_{H}} R_{H}(r) F\left(L, K_{H}\right)-\rho K_{H}
$$




\begin{tabular}{|c|c|c|c|}
\hline CBD & Central Business District, where firms are located & $r$ & distance from CBD \\
\hline$q$ & housing service per household & $h(r)$ & housing service density \\
\hline$z$ & composite goods & $k_{H}$ & housing capital density \\
\hline $\operatorname{Land}(r)$ & land surface at distance $r$ & $K_{H}$ & housing capital stock \\
\hline$n(r)$ & density of households at distance $r$ & $T(r)$ & transportation costs \\
\hline$c$ & consumption per capita & $r_{f}$ & city radius \\
\hline$R_{H}(r)$ & unit housing service rent & $R_{a}$ & agricultural land rent \\
\hline$H(r)$ & housing service at distance $r$ & $N$ & number of households \\
\hline$U(z, q)$ & utility function of a household & $u$ & utility level \\
\hline$x^{*}(r)$ & optimal capital to land ratio & $\rho$ & interest rate \\
\hline$F(K, L)$ & housing service production function & & \\
\hline
\end{tabular}

Table 1

Nomenclature for the traditional Von Thuenen model.

$$
\begin{aligned}
& k_{H}(r)=\frac{K_{H}}{L}(r)=\operatorname{argmax}\left[R_{H}(r) F(1, x)-\rho x\right] \\
& H(r)=\operatorname{Land}(r) \cdot F\left(1, k_{H}(r)\right) \\
& R_{H}(r)=0 \text { pour } r \geq r_{f} \\
& N=\int_{0}^{r_{f}} H(r) / q(r) d r
\end{aligned}
$$

A classic result of urban microeconomics (Fujita, 1989) is that if available land $\operatorname{Land}(r)$ is continuous and positive for all $r>0$, and if the consumption per capita $c$, the number of inhabitants $N$, transportation costs $T(r)$ and the interest rate $\rho$ are given, then Eqs. (1) to (7) define a unique utility equilibrium level $u^{*}$, homogenous in the whole city.

In Section 3, this framework is adapted so as to represent realistic urban dynamics.

\section{Non Equilibrium Dynamic Urban Model}

The monocentric model has been mostly used to explore the characteristics of long run equilibriums. The existence of urban stationary equilibriums, however, is questionable: in cities, some important economic variables vary in the 
short run, while other features of the city cannot adapt rapidly to changing conditions. Income and transportation costs, for example, evolve much more rapidly than housing infrastructures, which need several decades to be replaced. It is, therefore, very likely that the history of urban systems cannot be analyzed as a succession of stationary states, but requires a non stationary approach: assessing the effects of a policy that is supposed to change the city requires to account for the existence and specificities of transitions.

We propose here a model that is able to capture the dynamics of urban systems, and the importance of infrastructure and behaviors inertia. At the microeconomic level, we identify four key mechanisms that drive urban dynamics, and describe them in the following sections. Moreover, we ensure that NEDUM takes the main macroeconomic feedbacks into account.

A complete description of the model, with the full set of equations, is available in Appendix A. The nomenclature is summarized in Tab. 2, and presents the new variables that were not in the traditional monocentric model.

\subsection{Households behavior}

We assume that households respond to a given rent curve $R_{H}(r)$ in two ways:

- Households living at location $r$ adjust their housing service consumption per capita, so as to increase their utility level $u(r)$ : taking rent level $R_{H}(r)$ as given, households increase or decrease the size of their flats so as to equalize the marginal utility of housing service consumption and composite goods consumption. Adjustment in housing service consumption per capita is also done through changes in the size and composition of households, e.g. through changes in collocation practices, or changes in the age at which children leave their parents' home.

- Households can change locations: households living at location $r$ may choose to stay, or to move to another location $r^{\prime}$, while households living at other locations may move to location $r$. We assume that households decide to move by comparing local utility levels $u(r)$ with the average utility level $\bar{u}$ throughout the city: households living at locations where $u(r)<\bar{u}$ are attracted to places where $\bar{u}<u(r)$.

The processes considered here are, of course, active in parallel : changes in flat sizes occur simulatenously withy location changes, when households move from one flat to another. The changes are physically constrained by the characteristics of housing service supply: households can move only if there are unoccupied flats at their desired location; they can increase the size of their flats only if there is a local excess of housing service supply. These two mechanisms are the basis of local changes in demand for housing service (see Section 3.2). 
Most importantly, moves of households and changes in the sizes of flats cannot happen instantaneously, for instance because it takes time to find a new place to live. The respective inertias of these mechanisms are accounted for by specific characteristic timescales $\tau_{q}$ and $\tau_{n}$. The intensity of these mechanisms depend in each case on the increase in utility level that households expect from these evolutions: the higher is the relative difference between $u(r)$ and $\bar{u}$ for instance, the more numerous are households willing to move to location $r$.

\subsection{Rent curve dynamics}

Rent level $R_{H}(r)$ changes in reaction to local supply of housing service $H(r)$ on the one hand, and local demand of housing service on the other hand: this demand is expressed by the number of households $n(r)$ living at this location, and consuming an amount of housing service $q(r)$, and by the number of households willing to move to or from this location:

- The rent level decreases if local demand is inferior to local supply, that is, if existing buildings are not fully occupied.

- If buildings at location $r$ are fully occupied, rent levels increase if households living there want to increase their consumption of housing service, or if there are households living at other location that are willing to move at location $r$.

The orders of magnitude of these evolutions are determined by the relative difference between local demand and supply of housing service. Moreover, we assume that, for institutional reasons, housing rents do not clear the housing market instantaneously ${ }^{3}$. The inertia of rent levels evolution is characterized by the timescale $\tau_{R}$.

\subsection{Capital and investments}

In urban systems, buildings depreciate, and are renewed or constructed in reaction to rents and local demand for housing. These investments have a cost, and can have a crowding-out effect on other investments.

We add, therefore, to the monocentric model a description of capital stock evolutions in the housing and productive sectors. Investments are directed towards these two sectors. In the housing sector, investments are directed towards specific locations. The interest rate clears instantaneously the financial capital market. This macroeconomic feedback allows for the description of crowding-out of productive investments by housing investments.

$\overline{3}$ For instance, in France, rents are strongly regulated over 3-year periods. 
Since construction takes time (Kydland and Prescott, 1982), financial investments are transformed into productive units or into buildings with a time lag. In each sector, the timescales of this transformation of pending investments into effective investments are respectively $\tau_{k}$ and $\tau_{h}$.

\subsection{Specific functional forms and calibration}

In Appendix B, we show that, under standard conditions on general functional forms, NEDUM has one and only one stationary state, which recovers the classic equilibrium of the Von Thuenen framework. In the present section, we calibrate the model and explore the properties of NEDUM for a circular city, by adopting classic Cobb-Douglas functional forms for the utility function, the housing service production function, and the composite goods production function. We assume the generalized commuting costs to increase linearly with distance from the CBD. We reproduce in Appendix B the calculations that describe this stationary equilibrium with the specific functional forms we adopt.

We calibrate separately the set of parameters that determines the equilibrium state, and the set of parameters that only concerns the dynamics.

Equilibrium: the parameters of our model are calibrated so that, at equilibrium, it reproduces the characteristics of Los Angeles County. Of course, such a calibration is rough, if only because the L.-A. economy is open, while we do not take into account investment coming from or going outside L.A.. In 1999, 4.3 millions workers were living in this city, and they earn a $\$ 20700$ yearly per capita income (data U.S. Census Bureau 1999). The transportation price is calibrated using 1999 gasoline prices (i.e. 32 cents per km on average, data American Automobile Association 1999). Concerning the utility function, we chose $\beta$ so that housing expenditures represent $30 \%$ of households budget at equilibrium. Concerning macroeconomic feedbacks, we used for calibration the aggregate American investment rates. This lead us to an investment rate $s=19 \%$ (data Bureau of Economic Analysis 2006). We carried out systematic sensitivity analysis on these parameters, and found that the qualitative results presented in the remainder of this paper are quite robust.

Dynamics: calibration of parameters $\tau_{n}, \tau_{R}$ and $\tau_{q}$ is particularly difficult. We found, however, that their values, as long as they remain in a reasonable range of values, do not modify the qualitative results nor the orders of magnitude of the quantitative results of our paper. Typical values are explored: we consider $\tau_{n}=\tau_{R}=\tau_{q}=\tau=10$ years. These long response times account for the net flows in the city, and not for all individual moves or changes. A systematic sensitivity analysis was carried out with $\tau$ comprised between 3 years and 
20 years. We show some of these sensitivity analysis below. The results are also robust with respect to changes in $\tau_{k}$ and $\tau_{h}$, that can be approximated in an easier way, since they correspond to the construction duration of production units and buildings.

\section{Dynamic analysis of a shock on transportation costs}

In this section, we explore the effects of a shock on transportation prices. We assume that the initial state of the city (time $t=0$ ) is the stationary equilibrium described in Appendix B. Variables in the initial (resp. final) state are noted with a subscript " $i$ (resp. " $f$ "). We assume that in three years, starting at time $T$, transportation costs undergo a $50 \%$ increase, jumping from $p_{i}$ to $p_{f}=1.5 p_{i}$. We then use NEDUM to investigate how the city reacts to this shock.

In our numerical simulations, the city converges towards the stationary equilibrium corresponding to transportation costs $p_{f}$. This convergence, as is shown below, is very slow, and its slowness is a robust feature of our numerical simulations. Most importantly, our simulations allow to differentiate the situations with respect to space. At each location, we study the path followed after the shock by the economic system, from one stationary equilibrium to another.

\subsection{Average utility level}

The stationary equilibrium of our model verifies the classical results of comparative static analysis in urban economics (see for instance Wheaton, 1974). Following the shock in transportation costs, hence, the city concentrates towards the CBD. Rents increase near the CBD, and decrease at the outskirts. In the long run, consumers' utility decreases: after 150 years, average utility level $\bar{u}_{150}$ is $16 \%$ lower than initial utility $\bar{u}_{i}$ (see Fig. 1 ). At this date, the final equilibrium has almost been reached.

During the transition period, housing is not adapted to the new economic conditions, and the situation of the city inhabitants is significantly worsened, compared with the final stationary equilibrium. Figure 1 shows that, 25 years after the shock, the aggregate utility level in the city reaches a level which is $27 \%$ lower than the initial utility, and $12 \%$ lower than the final one. The order of magnitude of transition impacts and the length of this period are quite robust to changes in the timescales $\tau_{n}$ and $\tau_{q}$, which are successively divided by 2 in Fig. 1: in all cases, the average utility level in the city stays under $80 \%$ of its initial level for approximately 60 years. A division by 2 of 


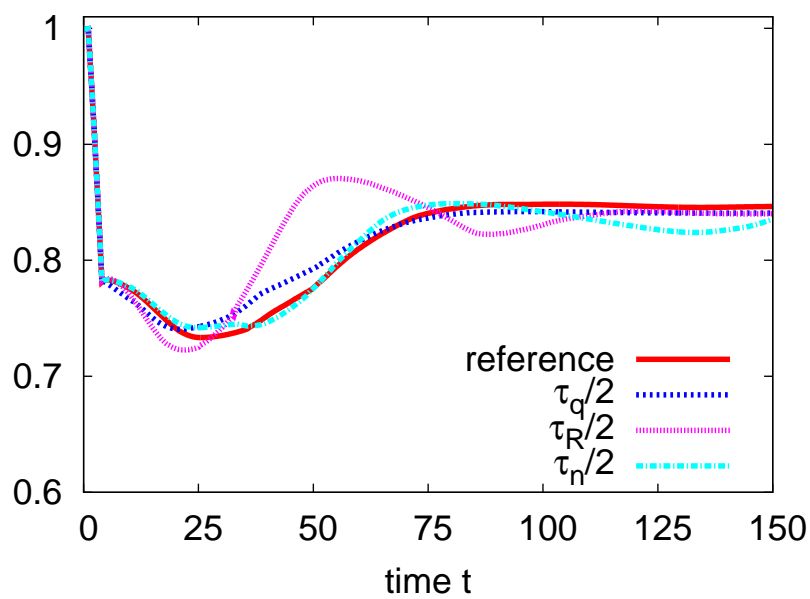

Fig. 1. The evolution of $\bar{u}$ with respect to time. Each curve corresponds to different values of the timescales $\tau_{n}, \tau_{R}$ and $\tau_{q}$ (index $\bar{u}=1$ at time $t=0$ ).

the timescale $\tau_{R}$ has a slighlty different effect on the dynamics of the system: the average utility level goes faster above the $80 \%$ value, though the length of the transition is roughly the same.

The aggregate effects of a shock on transportation costs, indeed, stem from the interaction of microeconomic behaviors: facing new, higher transportation costs, people want to move closer to the CBD. Before the moves actually occur, rent levels and flats' sizes have to change to create room for inhabitants willing to move. Over the longer term, the density of housing service supply (of which the height of the buildings is a good proxy) will also be adapted to the new conditions.

Simulations with NEDUM show that the duration of the transition depends mostly on the extent to which housing infrastructure is ill-located: it takes a long time to collect and direct great quantities of capital towards the locations where new housing is needed. In a non anticipating framework, actors need a signal before expressing a new demand. The reaction of the market depends on:

- the time scales $\tau_{n}, \tau_{R}$, and $\tau_{q}$;

- parameters of the housing procution function $F(K, L)$, and the investment capacity of the whole economic system.

The timescales of the latter mechanism are driven by the Cobb-Douglas housing production function $F(K, L)$, the composite goods production function $Y(K, N)$, and the investment rate $s$. Providing adequate housing investment is much longer than the phenomenons driven by $\tau_{n}, \tau_{R}$, or $\tau_{q}$, and is, therefore, the mechanism responsible for the 30 years period. In the sections below, we 
enter the details of this ill-adaptation through time and space.

\subsection{Spatialized adaptation}

Initial location choices of households were subjected to initial transportation price $p_{i}$ and the initial rent levels throughout the city (see Eq. (2)). As transportation costs get higher, households are willing to move closer to the CBD to spend less on transportation. As a consequence, rent levels go higher in central locations. The left panel of Fig. 2 shows that 5 years after the shock, rents close to the $\mathrm{CBD}$ have already increased, and are close to their final level. However, rents have not changed far from the CBD: population density has not changed, even though households are willing to leave these locations. They have, indeed, to stay there since there is no unoccupied flat yet in the central buildings to allow their moves (see the right panel of Fig. 2).
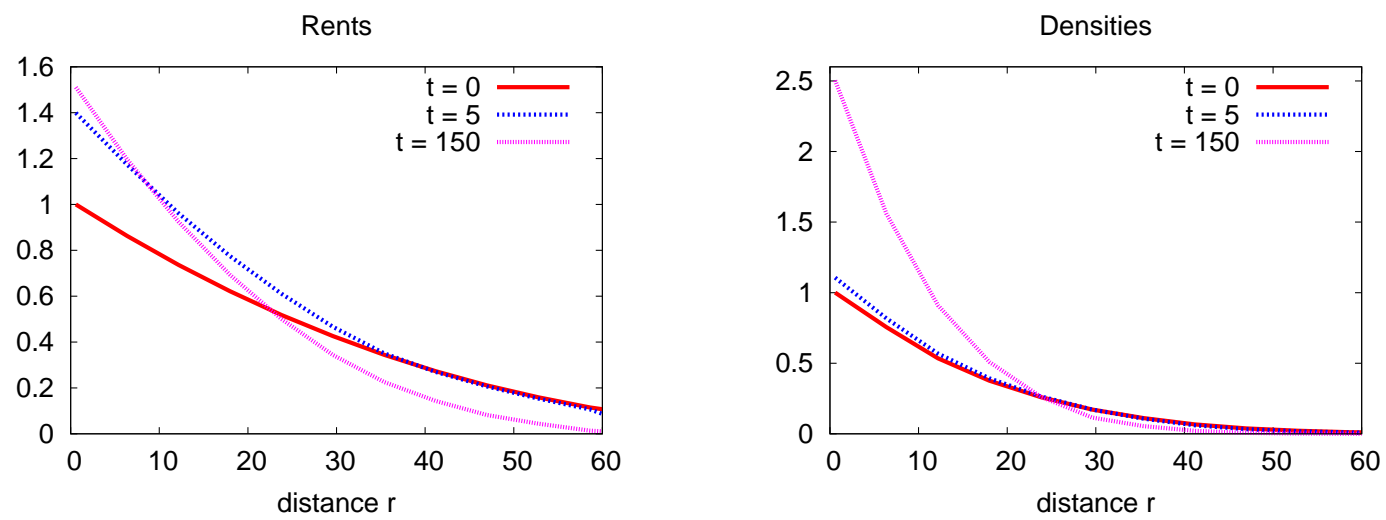

Fig. 2. Left: rent curves $R_{H}(r)$ before the shock, 5 years after the shock, and 150 years after the shock (index $R_{H}(0)=1$ before the shock). Right: density curves $n(r)$ before the shock, 5 years after the shock, and 150 years after the shock (index $n(0)=1$ before the shock).

Of course, rent levels feedback to the rest of the system:

- Housing construction is enhanced in the city center.

- Rent levels also constrain households living close to the CBD to reduce their housing service consumption per capita, thus letting more space for households willing to move in.

Figure 3 shows that 15 years after the shock, many moves have occurred. At this date, since households have left the outskirts of the city, rents are almost at their final level at all locations. The dynamic of rents and moves, therefore, is almost completed in 15 years.

It is not the case, however, for construction. Construction demands a large amount of capital, and it takes time for the housing capital stock to reach 
Rents

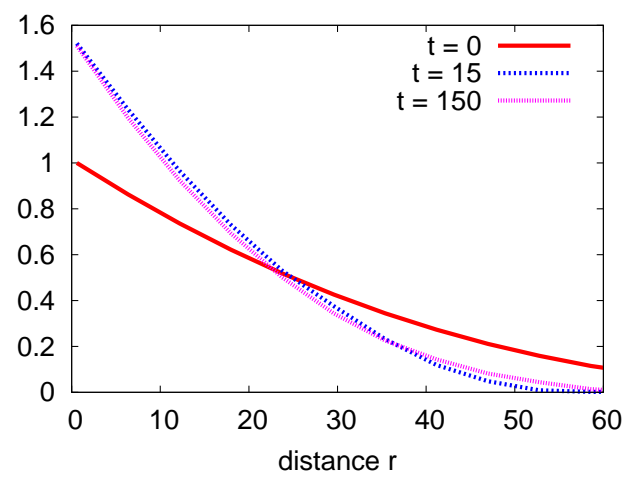

Densities

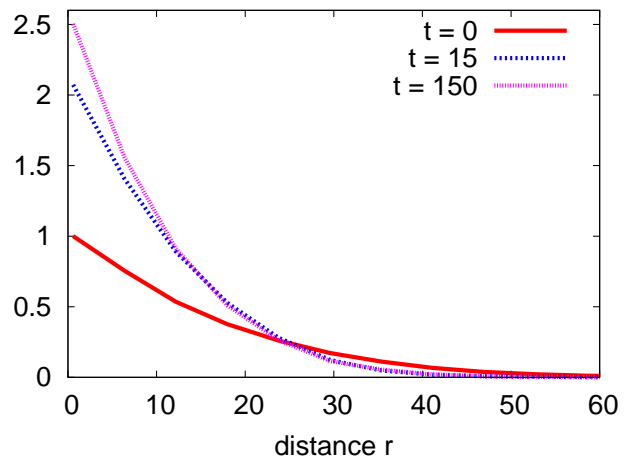

Fig. 3. Left: rent curves $R_{H}(r)$ before the shock, 15 years after the shock, and 150 years after the shock (index $R_{H}(0)=1$ before the shock). Right: density curves $n(r)$ before the shock, 15 years after the shock, and 150 years after the shock (index $n(0)=1$ before the shock).

the appropriate level. As a consequence, compared to the final equilibrium, the supply of housing service is still not concentrated enough 15 years after the shock, which explains the low level of average utility during the transition period: housing service consumption per capita depends on housing supply, which has not reached its final level as long as housing service supply is not at a stationary state.

\subsection{Redistributive consequences}

The transition is very differentiated with respect both to time and space: we considered a stationary equilibrium as the initial state of our system. In this situation, all households earn the same income, and they reach the same utility level: $u(r)=\bar{u}$ at all locations. After the shock, however, $u(r)$ is not the same anymore throughout the city. Households located far from the CBD have to put up with very high transportation costs, but do not see their rents decrease immediately. The shock, therefore, impacts them strongly. The left panel of Fig. 4 shows that, 10 years after the shock, those living at $50 \mathrm{~km}$ from the CBD can lose up to $38 \%$ compared to their initial utility level.

Meanwhile, households living closer to the CBD use less transportation for commuting. The initial losses are, therefore, not so big for them, amounting to merely $4 \%$ of the initial utility level 10 years after the shock. Later, they get worse as rents increase because of the increased demand at their location. Of course, all utility levels converge in the long run towards a common value. This convergence is very slow, as illustrated by the left panel of Fig. 4; this slowness is partly due to the fact that, as utility levels $u(r)$ get closer to $\bar{u}$, incentives to move diminish (see Eq. (A-21)). 
Specific utility levels

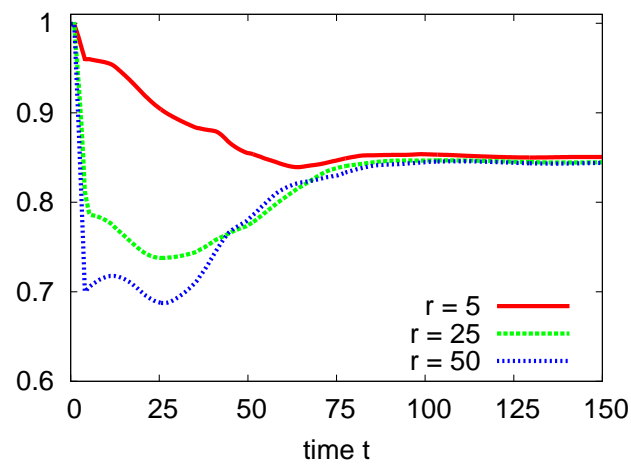

Redistributive effects

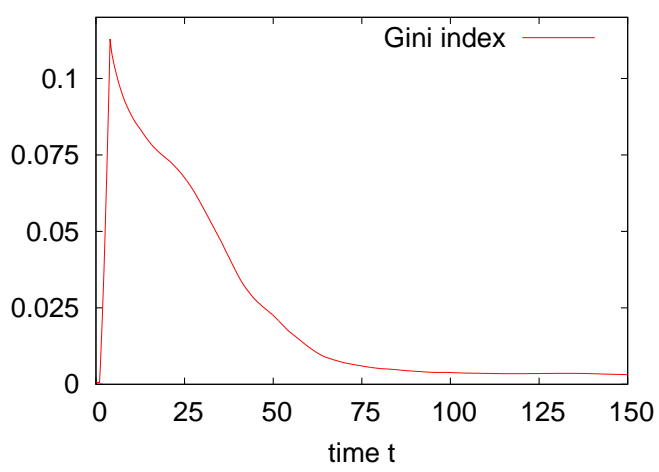

Fig. 4. Left: evolution of utility levels with respect to the time, at locations $5 \mathrm{~km}$, $25 \mathrm{~km}$, and $50 \mathrm{~km}$ in the city (index $u=1$ before the shock) for 150 years after a shock in transportation costs. Right: evolution of the gini index characterizing inequalities of utility levels in the city.

The redistributive effects are far from negligible. The right panel of Fig. 4 shows that the Gini index jumps from 0 to 0.12 right after the shock. This difference means that a part of the population would be very strongly impacted by the shock, since these jumps of the Gini index are very important. For instance, according to Watkins et al. (2006), at the national levels, Gini indexes of the US, the UK, and in France, are respectively $0.40,0.36$, and 0.32 : those three countries have Gini indexes that are comprised in a 0.08 wide range of values. ${ }^{4}$ In our simulation, during the transition period, the Gini index decreases as the adaptation mechanisms (moves, changes in the sizes of flats, changes in the rent levels, construction) enter into action: with our calibration, it stays above 0.02 during 50 years. After this transition, the decrease of the Gini index back to zero is slower, for the reason already mentioned (i.e. the form of the weighting functions).

\subsection{Crowding-out effect and macroeconomic feedbacks}

The need to invest into reconstruction enhances the negative impacts of the transition through crowding-out effects: as rents close to the CBD increase, housing service production gets more profitable, and investments are directed towards the production of housing service at those locations. As a consequence, capital is more sought after by investors, and the interest rate increases by almost 0.25 points (see the right panel of Fig. 5). On the long run, however, the rate of interest asymptotically returns to its initial level. Meanwhile, there is a crowding-out effect of productive investments by housing investments, and 36 years after the shock, the production of composite goods has decreased by

4 Of course, Gini indexes can be far higher. Brazil's Gini index, for instance, reaches 0.58 . 
$1.2 \%$ (see the left panel of Fig. 5). Given its timing, and even though the aggregate product returns to its initial level in the long run, this effect is important: household income decreases in the same time as they face a shock on transportation costs.

It is noteworthy that, in the real world, the same mechanism that increases the cost of capital should also apply to labor. Following increased profitability of housing service production, wages go up in this sector, inducing the workers to switch from the productive sector to the construction sector. Comparable mechanisms occur after a disaster, when reconstruction needs make the wage of qualified workers increase by up to $40 \%$. This phenomenon would enhance the cost of crowding-out effects. For simplicity's sake, however, we assumed that wages were fixed, and that all workers were employed in the productive sector.
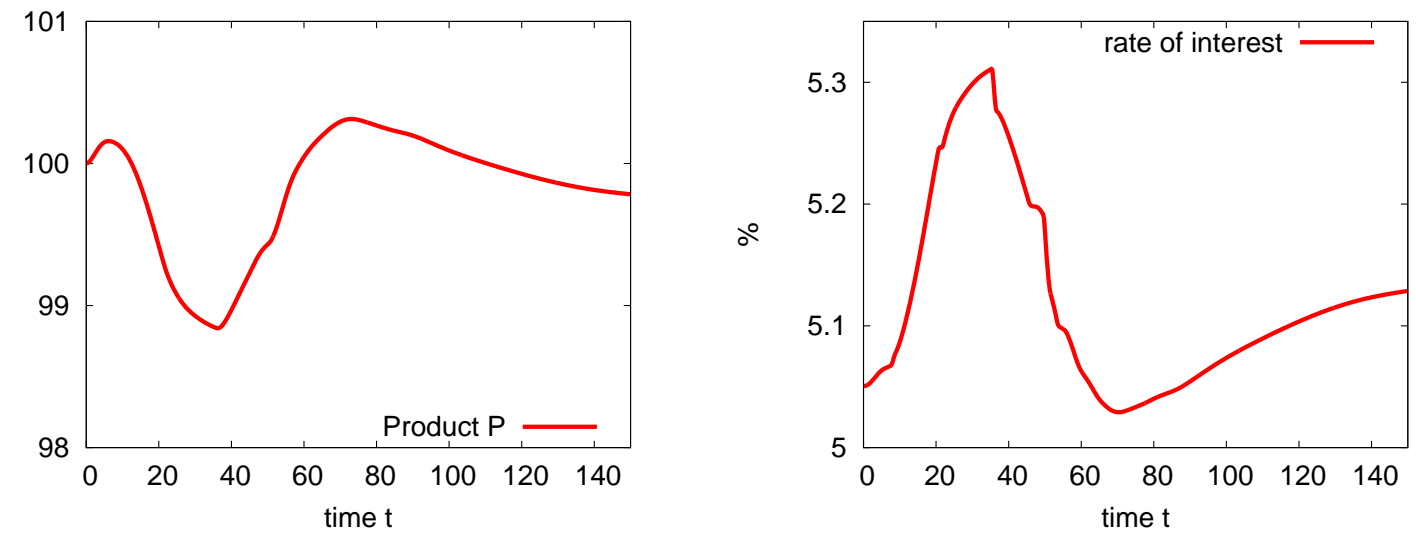

Fig. 5. Evolution of the economic output with respect to time (index $Y=100$ before the shock), and of the rate of interest $\rho$.

\section{Sending a signal-price: when late is too late}

In this section, we assume that the government has decided to set a given tax level on transportation in year 2050. This situation may arise for instance in the framework of climate negotiation, if international agreements are reached on the marginal cost of carbon emissions and its internalization through a carbon tax. We consider year 2050 because it may be the case that marginal carbon prices reach high level by then, and because it is far away in time. It allows, therefore, to analyze the importance of the implementation rythm of the signal-price over long periods of time. 


\subsection{Early versus delayed action}

We assume that the policy works as follows:

- when a transportation tax is implemented, workers have to pay an increased transportation cost $T(r)=(p+\tau) r$. The product of the tax, namely $\pi=\tau \int_{0}^{r_{f}} n(r) r d r$, is lump-sum redistributed to the workers, who use it for consumption.

- The government chooses to increase linearly the tax level $\tau(t)$ from its initial level $\tau_{i}=0$ to its final level $\tau_{f}=p / 2$. The slope of this increase depends on the year the government chooses to start the implementation of transport taxation.

We study implementation periods that run from 60 years down to 0 . Thus, latest implementation begins in year 2050, while earliest implementation begins in year 1990. We consider such an early anticipation, since high levels of transport taxation already exist in some countries, even though in year 1990 those tax levels were not related to climate policies.
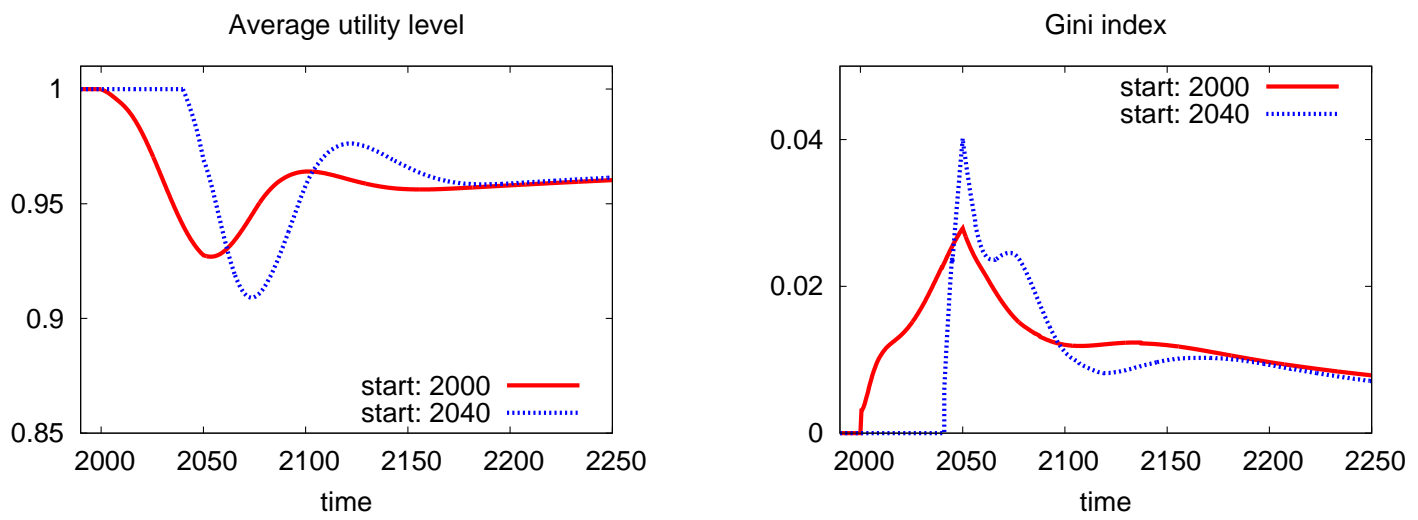

Fig. 6. Left: average utility level with respect to time, for policies starting in year 2000 and year 2040 (index $u=1$ in year 1990). Right: Gini index with respect to time for policies starting in year 2000 and year 2040.

We estimate these various policies over the long run, namely until year 2250. This very long period is necessary since the return to the stationary equilibrium is very slow. At this date, different paths have lead to almost identical situations, and that the situation of the urban system is almost independant of the implementation policy (see Fig. 6).

\subsection{Welfare losses}

It is difficult to predict a priori which type of implementation (early or late) is likely to be the less costly. Smooth implementation makes inertia in the urban 
system less detrimental, but does not suppress completely its effects. Early implementation induces the system to converge sooner towards a stationary state that is under-optimal, at least if the impacts of carbon emissions on the environment and the economy (i.e. the benefits of the policy) are not taken into account. With this limited conception of welfare, an early implementation leads to welfare losses which are accumulated over a long period of time (as soon as the policy begins). Late implementation, on the other hand, induces more abrupt welfare losses, that are more concentrated in time. As an illustration, Fig. 6 shows the average utility level for a "smooth" policy, starting in year 2000, and an "aggressive" policy, starting in year 2040.

We compare these costs to a baseline scenario where no action is implemented at all. According to our simulations (see the left panel of Fig. 7, where each curve corresponds to different values of the model parameters), welfare costs can represent a $3.8 \%$ to $2.1 \%$ loss over the entire period. These relatively high losses are due to several factors, some of which are not taken into account in published assessments of mitigation costs (Weyant et al., 2006; Edenhofer et al., 2006): (1) consumers spend more money in transportation for a given commuting distance; (2) they live in smaller flats because of the higher burden from transportation costs; and (3) the preexisting spatial distribution of flats is ill-adapted during the transition.
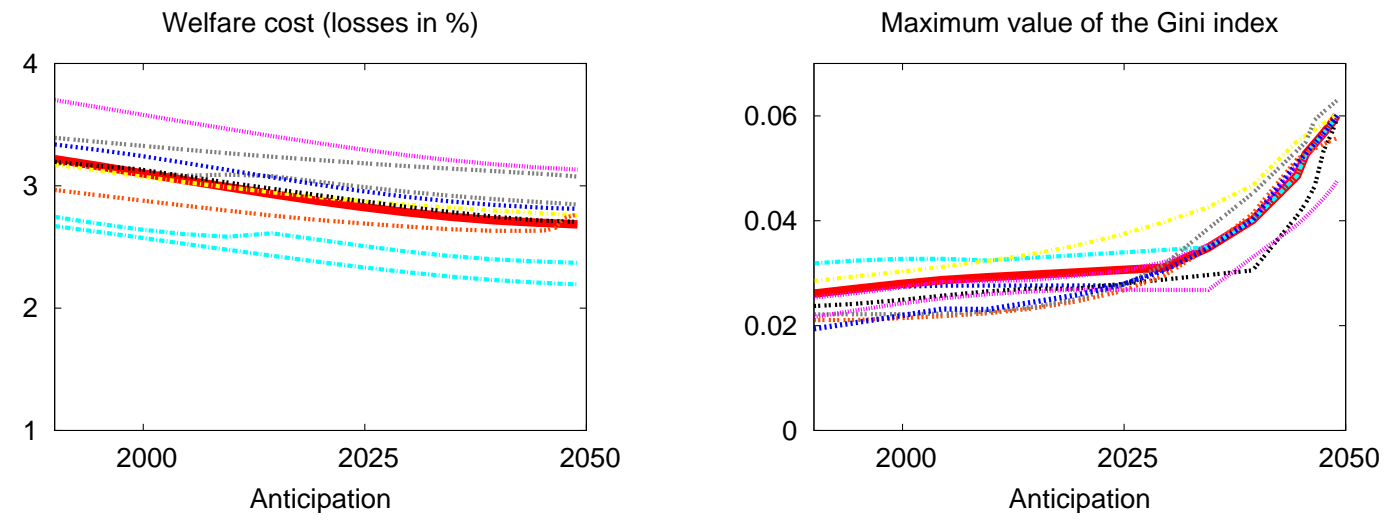

Fig. 7. On these two panels, each curve is related to a sensitivity test to a given parameter. We show curves corresponding to a wide range of values of $\tau_{R}, \tau_{q}, \tau_{n}$, $a$, and $\delta_{H}$. The curve corresponding to the central values of these parameters is the bold red curve. Left: the welfare costs of tax implementation, with respect to the year of implementation. Right: the maximum value reached by the Gini index during the transition, depending on the year of implementation.

Simulations show that welfare costs decrease when the action is delayed. Thus, the gains due to the neutralization of inertia effects are more than compensated by the increased losses due to the under-optimality of the final stationary state. Note, however, that this welfare assessment is not a cost-benefit analysis, since benefits are not taken into account. Meanwhile, we show here that, in assessing public policies, investigating aggregate effects is not enough, and the 
consequences in terms of inequality are also crucial.

\subsection{Redistributive effects}

The influence of early action on inequality is not ambiguous. The maximum value reached by the Gini index increases when implementation is delayed (see the right panel of Fig. 6 for an illustration).

Most importantly, redistributive effects are non-linear with respect to the year of implementation. On the right panel of Fig. 7, each curve corresponds to different values of the parameters we use in NEDUM. Each point of one of these curves shows, for a given year of implementation, the maximum value of the Gini index that will be reached during the whole following implementation period. For instance, if the tax is implemented in year 2020 and induces a maximum value of the Gini index 0.024, reached in year 2037 (see right panel of Fig. 6), then the point (2020; 0.024) belongs to the curve. We consider the maximum value of the Gini index since it is a good indicator of the redistributive impact of a policy.

Two features can be derived from the analysis of these redistributive effects.

- For all the sensitivity tests we performed, the maximum value of the Gini index remains very close to 0.02 as long as the implementation period begins before year 2015.

- This is clearly not the case anymore if the urban policy begins after year 2020: the spectrum of values of the Gini index gets larger, and increases rapidly. Late implementation, starting in year 2049, induces maximum values of the Gini index that range from 0.05 to 0.08 , which is clearly a major disruption of the social situation.

As a consequence, delaying the tax implementation from year 2015 to year 2049 induces a decrease in welfare losses by 1 point, but causes Gini index to reach a very high level. This is an equity vs. efficiency trade-off that cannot be easily resolved. For instance, using the tax product to compensate households living far from the center, rather than lump-sum distributing this product, would distort the signal being sent to households, and limit the efficiency of the policy. Indeed, the tax is meant to internalize the costs of carbon emissions and decrease mobility demand, which cannot be done without creating inequality. Doing so smoothly, however, reduces the redistributive shock. 


\section{Conclusion}

\subsection{Summary}

This paper presents a new model, NEDUM, as a support for urban dynamic and policy analysis. Without pretending to produce precise cost estimates, this model allows for the analysis of stylized dynamic effects, and the assessment of the orders of magnitude that are specific to transitions. We focus on the importance of inertia in infrastructures renewal, in households moves, in changes in the sizes of flats, and on stickiness of housing rents.

In the long run, the average utility level decreases since consumers spend more money in transportation, and live in smaller flats. In the short- and medium-run, we show that transitions are characterized by significant impacts on welfare: after a $50 \%$ shock in transportation costs, losses in average utility are significantly larger during the transition than in the long run stationary state. This is due mostly to the adaptation pace of the urban system to the new transportation costs. These inertia effects are worsened by the crowdingout effect of investments from the productive to the housing sector, following reconstruction needs: production of composite goods is decreased by as much as $1.2 \%$ during the transition. Taking into account these mechanisms may change significantly the assessment of GHG stabilization strategies, compared with published assessments, see e.g. Weyant et al. (2006) or Edenhofer et al. (2006).

Our study of a tax implementation shows, however, that it is possible to smooth out the impacts of transitions through time, but on the whole, this kind of early action does not necessarily compensate the fact that welfare losses begin earlier, as implementation starts. Utility changes are strongly differentiated in time and space, and transition is a significant phenomenon (lasting approximately 30 years in our examples), that cannot be ignored, especially from the redistributive point of view.

Because of inertia, changes in urban transportation systems have significant redistributive effects. The reason is that the demand for transportation is differentiated in space. Thus, location matters: even though all utility levels in our imaginary city are eventually equal, there exist non-trivial paths between the utility levels immediately after the shock and the final utility $u_{f}$. Following an increase in transportation costs, consumers living far from the CBD have a strong burden to cope with, and cannot move immediately to locations where their utility level would be higher, because housing is not available close to employment centers.

The magnitude of the redistributive effects is directly related to the aggres- 
siveness of the change, i.e. the amplitude of the modifications, and their pace. Considering the implementation of a carbon tax, for instance, we find that there exists a trade-off between equity and efficiency, and that the vulnerability of urban systems to redistributive effects increases non-linearly as the implementation duration is reduced.

As a consequence, delaying implementation of a carbon tax by 35 years may reduce welfare losses by 1 point of percentage, but might also cause significative redistributive changes. In fact, if the tax is intended to reduce greenhouse gas emissions, early implementation induces also earlier reductions : this aspect increases the efficiency of the tax compared to our simulations, but does not change the redistributive effects. As a consequence, assessing the effects of an urban policy requires the taking into account of all these evolutions leading to the final equilibrium, under the constraints imposed by our general equilibrium framework.

\subsection{Discussion}

It is noteworthy that most policy-makers are elected for short timescales (typically, inferior to 5-10 years). Hence, even if they do take into account the long term benefits of their decisions, they are very sensitive to short-term effects. Considering a carbon tax implementation for instance, they would be reluctant to implement transportation policies with such kind of negative transition effects, especially when some households are particularly impacted. Therefore, regarding the design of urban or climate policies, inequalities are likely to be at least as important implementation obstacles as welfare losses. If a carbon tax is considered as necessary in year 2050, waiting until 2049 to implement it is a dangerous solution. It is important, therefore, that international negotiations take this aspect into account, ensuring that smooth implementation path of the signal price are favored.

Of course, things are more complex in the real world, and transition effects depend on the specific features of each city. NEDUM is only a first step towards a dynamic assessment of urban changes, and this first version has several limitations. Of course, the usual limitations involved in an analysis based on the classic Von Thuenen model are also present ${ }^{5}$, though it seems at first view that they would interfere only marginally with our results. Main differences with the real world are the existence of several employment centers, the taking into account of congestion in transportation systems, and the co-existence of several transportation systems. There are, however, other limitations to our

5 Except for the most important macroeconomic feedbacks, which are present in NEDUM. 
model, that may be more important, and constitute a program for future research.

The first question NEDUM should be able to tackle next is the importance of anticipations: our assumption of agents' myopia was an extreme one. In the real world, agents have expectations, either false or true, and these anticipations influence the functioning of the whole system as well as the pace of changes. It is particularly important to include these aspects in NEDUM so as to be able to analyse commitment problems on behalf of the governement, and the possibility of time-inconsistent taxation patterns.

Second, another important dynamic aspect is absent from our economy: population change and economic growth has not been taken into account. Clearly, growth modifies the impacts of changes in the transportation system, since economic conditions evolve continuously. Though the value of welfare losses would be impacted, it is likely, however, that the sensibility of redistributive effects to the pace of changes would not be very different than in a world without growth: spatial discrimination would still exist.

Third, destruction costs are likely to be important, and interplay with depreciation rates of buildings, which are very different in each country. We did not take into account an accelerated rate of building turnover. It is likely that this timescale influences strongly the vulnerability of urban systems to changes, and their capacity for adaptation.

Also, all households do not earn the same income in the real world. It is likely that in American cities, where low income workers usually live in the center while rich households live at the outskirts, the Gini index would reach a different level, and may even go down. In European cities, where city centers are mostly inhabited by rich households, the Gini index would probably go even higher. In both types of cities, anyway, important redistributive effects would occur, and their taking into account should be a priority in policy design.

\section{References}

Alonso, W., 1964. Location and Land Use. Harvard University Press.

Anas, A., 1978. Dynamics of urban residential growth. Journal of Urban Economics 5, 66-87.

Brueckner, J. K., 2000. Economics of Cities - theoretical perspectives. Cambridge University Press, Ch. 7, pp. $263-289$.

Capozza, D. R., Helsley, R. W., 1990. The stochastic city. Journal of Urban Economics 28, 295-306.

Collier, U., Loefstedt, R. E., apr 1997. Think globally, act locally? local climate 
change and energy policies in sweden and the uk. Global Environmental Change 7 (1), 25-40.

Edenhofer, O., Lessmann, K., Kemfert, C., Grubb, M., Kohler, J., 2006. Induced technological change: Exploring its implications for the economics of atmospheric stabilization: Synthesis report from the innovation modeling comparison project. The Energy Journal Special Issue n4 27, 57-108.

Fujita, M., 1989. Urban Economic Theory — Land Use and City Size. Cambridge University Press.

Gusdorf, F., Hallegatte, S., 2007. Behaviors and housing inertia are key factor in determining the consequences of a shock in transportation costs. Energy Policy 35, 3483-3495.

Kydland, F. E., Prescott, E. C., nov 1982. Time to build and aggregate fluctuations. Econometrica 50 (6), 1345-1370.

Mills, S. E., 1967. An aggregative model of resource allocation in a metropolitan area. The American Economic Review 57 (2), 197-210, papers and Proceedings of the Seventy-ninth Annual Meeting of the American Economic Association.

Muth, R. F., 1969. Cities and Housing - The Spatial Pattern of Urban Residential Land Use. The University of Chicago Press.

Rotmans, J., Hulme, M., Downing, T. E., jun 1994. Climate change implications for europe - an application of the escape model. Global Environmental Change 4 (2), 97-124.

Srinivas, H., oct 2000. Focusing on the real environmental culprits urban areas unu's city inspirations initiative. Global Environmental Change 10 (3), 233236.

Tulkens, P., Tulkens, H., june 2006. The White House and the Kyoto Protocol: Double Standards on Uncertainties and their Consequences, FEEM Working Paper, available on line at http://www.feem.it.

Von Thuenen, J. H., 1826. Der Isolierte Staat in Beziehung auf Landwirtschaft und Nationaloekonomie. Perthes.

Watkins, K., Carvajal, L., Coppard, D., Fuentes, R., Ghosh, A., Giamberardini, C., Johansson, C., Seck, P., Ugaz, C., Yaqub, S., 2006. Human Development Report: 2006. Tech. rep., United Nations Development Programme. Weyant, J. P., de la Chesnaye, F. C., Blanford, G. J., 2006. Overview of emf-21: Multigas mitigation and climate policy. The Energy Journal Special Issue n3.

Wheaton, W. C., 1974. A comparative static analysis of urban spatial structure. Journal of Economic Theory 9, 223-237. 


\section{A Appendix: The Non Equilibrium Dynamic Model}

This appendix sets the formal representation of the mechanisms we describe in Section 3. Table 2 summarizes the nomenclature for the new variables that were not presented in the traditional Von Thuenen model.

\begin{tabular}{||ll||ll||}
\hline$Y(K, N)$ & composite goods production function & $\widetilde{I_{f H}}$ & financial investment in housing capital \\
$\delta_{K}$ & discount factor of the productive capital & $\widetilde{I_{f K}}$ & financial investment in productive capital \\
$\delta_{H}$ & discount factor of the housing capital & $S_{H}$ & stock of pending investments \\
$\rho$ & capital price & & in housing capital \\
$\theta$ & tax level & $S_{K}$ & stock of pending investments \\
$\pi$ & tax product & in productive capital \\
$L I$ & Land Income & $I_{H}$ & physical investment in housing capital \\
$\tau_{R}$ & timescale of rent evolution & $I_{K}$ & physical investment in productive capital \\
$\tau_{q}$ & timescale of the evolution of & & timescale of the evolution of \\
& housing service per capita & pending housing investments \\
$\tau_{k}$ & timescale of the evolution of & timescale of moves \\
& pending productive investments & & \\
\hline
\end{tabular}

Table 2

Nomenclature: new variables introduced in NEDUM.

\section{A.1 Capital, and investment drivers}

\section{A.1.1 Production and consumption}

While the income in Section 2 is exogenous, we specify here a production function $Y$, the inputs of which are labor $N$, and productive capital $K$ :

$$
Y=Y(K, N)
$$

A constant part of the product is saved and shared between financial productive and housing investment (respectively $\widetilde{I_{f K}}$ and $\widetilde{I_{f H}}$ ), while the other part $c$ is used by households for consumption. For simplicity reason, we assume that land is publicly owned: land incomes $L I$ are collected by the government, and lump-sum redistributed to consumers, who use it for consumption.

$$
\begin{aligned}
& s \cdot Y=\widetilde{I_{f H}}+\widetilde{I_{f K}} \\
& c=(1-s) \cdot \frac{Y}{N}+\frac{L I}{N}
\end{aligned}
$$


Equilibrium in financial markets is ensured by the adjustment of the capital price, the interest rate $\rho$.

\section{A.1.2 Productive investments}

The variable $K$ is the capital stock in the productive sector, and $\delta_{K}$ is the depreciation rate of capital. Firms seek to maximize their profits, and have a myopic behavior: they make investment decisions as if they were in a stationary state equilibrium. This leads to the financial investment $\widetilde{I_{f K}}$ in the productive sector:

$$
\widetilde{I_{f K}}=\delta_{K} \cdot \arg \max _{K}\left[Y(N, K)-\left(\rho+\delta_{K}\right) \cdot K\right]
$$

Physical construction requires time (Kydland and Prescott, 1982); thus, financial investments are transformed into productive capital with a time lag, corresponding to construction duration. We set $S_{K}$ as the resulting stock of "pending investments" in productive capital. nd $I_{K}$ is the real physical investment, which evolves according to the following equations:

$$
\begin{aligned}
& \frac{d S_{K}}{d t}=-I_{K}+\widetilde{I_{f K}} \\
& I_{K}=\frac{1}{\tau_{k}} \cdot S_{K} \\
& \frac{d K}{d t}=-\delta_{K} K+I_{K}
\end{aligned}
$$

\section{A.1.3 Housing investment}

Housing is produced using land and capital ${ }^{6}$. The modeling of investments in the housing sector is based on the same principles that drive investment in the productive sector. A little complication is added, however, due to the fact that the location of housing investments is driven not only by interest rate, but also by rent levels, which vary with location (see how the density of available housing service is linked to housing capital stock in Eq. (5)).

Investors owning land at location $r$ are price-takers for rent levels and interest rate. They invest $I_{f H}(r)$ given by:

$$
I_{f H}(r)=\delta_{H} \cdot \arg \max _{K_{H}(r)}\left[R_{H}(r) \cdot F\left(K_{H}(r), \operatorname{Land}(r)\right)-\left(\rho+\delta_{H}\right) \cdot K_{H}(r)\right]
$$

\footnotetext{
${ }^{6}$ For simplicity's sake, labor is exclusively used for the production of the composite goods. This assumption is of course a limitation of the model: we chose to focus our analysis of crowding-out effects on capital rather than labor.
} 
This behavior leads to the aggregate demand for housing investment $\widetilde{I_{f H}}$ :

$$
\widetilde{I_{f H}}=\int_{0}^{r_{f}} I_{f H}(r) d r
$$

As for productive capital, there is a lag between financial capital $\widetilde{I_{f H}}$, and physically invested capital $I_{H}$, a lag given by $\tau_{h}$ that corresponds to the time required to achieve construction of buildings:

$$
\begin{aligned}
& \frac{d S_{H}}{d t}(r)=-I_{H}(r)+I_{f H}(r) \\
& I_{H}(r)=\frac{1}{\tau_{h}} S_{H}(r) \\
& \frac{d K_{H}(r)}{d t}=-\delta_{H} K_{H}(r)+I_{H}(r)
\end{aligned}
$$

\section{A.2 Households behavior}

\section{A.2.1 Housing service per household}

We assume that households permanently adapt their housing-service consumption to prices variation. We set $\tilde{u}(q, r)$ the utility level reached by households living at a distance $r$ from the CBD, as a function of housing service consumption $q$; at distance $r$ the amount of composite goods consumed is strictly dependant on housing choices: $z=c-T(r)-R_{H}(r) q$. We have, therefore, $\tilde{u}(q, r)=U\left(\left[c-T(r)-R_{H}(r) q\right], q\right)$. By using this function, we consider that households can adjust their level of housing service consumption so as to improve their utility.

Based on this relationship, at a given location, a change in housing service consumption per capita $\delta q$ induces a change in utility: $d \tilde{u}=\left(\frac{\partial \tilde{u}}{\partial q}\right) d q$. If at location $r, \frac{\partial \tilde{u}}{\partial q}>0$, it is indeed rational for those who live at this location to increase their consumption of housing service. Of course, an increase in housing consumption per capita is authorized if and only if such an increase is physically possible, i.e. if there is available housing at location $r$. Thus, the dynamics of $q(r)$ is given by:

$$
\frac{d q}{d t}(r)=\left\{\begin{array}{l}
\frac{1}{\tau_{q}} g\left(\frac{\partial \tilde{u}}{\partial q}\right) \text { if } \psi(r)>0 \\
0 \text { if } \psi(r)=0
\end{array}\right.
$$


where $g(x)$ has the same sign as its argument $x$, and increases with respect to $x$; moreover, $\psi(r)$ represents the number of unoccupied flats at location $r$, calculated as:

$$
\psi(r)=\frac{H(r)}{q(r)}-n(r)
$$

\section{A.2.2 Moves throughout the city}

Consumers have the possibility to move and change location across the city. They are driven by the utility levels $u(r)$ that characterize locations. We set $\bar{u}$ as the average utility level of consumers throughout the city. At a given location $r$, two cases can arise, depending on the utility level $u(r)$.

- If $u(r)<\bar{u}$, households living at location $r$ are willing to leave towards other locations. We set $m^{-}(r)$ as the number of households that are willing to move out:

$$
m^{-}(r)=n(r) w^{-}(u(r), \bar{u})
$$

In Eq. (A-10), the willingness to move from a location is larger if the gap between $u(r)$ and $\bar{u}$ is large, and $w^{-}$is a "weight" function depending on this gap: $\left.w^{-}(u(r), \bar{u}) \in\right] 0,1\left[\right.$, and $w^{-}$is a function that increases with respect to $\left|\frac{\bar{u}-u(r)}{\bar{u}}\right|$.

- If $u(r)>\bar{u}$, then households located at other locations are willing to move towards location $r$. We set $m^{+}(r)$ as the number of unoccupied flats at location $r$ that are attracting households living at an other location. This attractiveness is larger if the gap between $u(r)$ and $\bar{u}$ is large:

$$
m^{+}(r)=\psi(r) w^{+}(u(r), \bar{u})
$$

In Eq. $\left.(\mathrm{A}-11), w^{+}(u(r), \bar{u}) \in\right] 0,1\left[\right.$, and $w^{+}$increases with respect to $\left|\frac{u(r)-\bar{u}}{\bar{u}}\right|$.

Based on Eqs. (A-10) and (A-11), the aggregate demand for moves and the aggregate supply of attractive, unoccupied flats, are given respectively by:

$$
\begin{aligned}
& \widetilde{D}=\int_{u(r)<\bar{u}} m^{-}(r) d r=\int_{u(r)<\bar{u}} n(r) \cdot w^{-}(u(r), \bar{u}) d r \\
& \widetilde{S}=\int_{u(r)>\bar{u}} m^{+}(r) d r=\int_{u(r)>\bar{u}} \psi(r) \cdot w^{+}(u(r), \bar{u}) d r
\end{aligned}
$$

Households move only if they can increase their utility level, and if there is available housing at their desired destination. However, there is a priori no 
reason why the demand for moves should equal the supply of available housing. The relationships giving the moves $\mu(r)$ meet these physical constraints:

$$
\mu(r)= \begin{cases}m^{+}(r) \cdot \min \left(1, \frac{\widetilde{D}}{\widetilde{S}}\right) & \text { if } u(r)>\bar{u} \\ -m^{-}(r) \cdot \min \left(1, \frac{\widetilde{S}}{\widetilde{D}}\right) & \text { if } u(r)<\bar{u}\end{cases}
$$

The variable $d(r)$ represents the number of households that are attracted by location $r$. It can be greater than the number of moves $\mu(r)$, since the demand may exceed the supply of unoccupied flats:

$$
d(r)=m^{+}(r) \cdot \frac{\widetilde{D}}{\widetilde{S}}
$$

In Eq. (A-15), the coefficient $\frac{\widetilde{D}}{\widetilde{S}}$ represents the number of "candidates" per unoccupied flat. If aggregate demand is smaller than aggregate supply, not all available housings will find an occupier. If aggregate demand is greater than aggregate supply, then there are more potential moves than available housings, and not all consumers willing to move will find a new housing.

The number of households living at location $r$ evolves according to the moves:

$$
\frac{\partial n}{\partial t}(r)=\frac{1}{\tau_{n}} \mu(r)
$$

\section{A.3 Rent curve dynamics}

In the classical Von Thuenen framework, housing market is at equilibrium thanks to the rent curve (cf. Section 2). It is not necessarily the case during transitions. In real life, rent levels are sticky for institutional and practical reasons. Thus, we define a dynamics for rents throughout the city, directed by supply and demand for housing service at each location: if the number $d(r)$ of households willing to move in is greater than the number of unoccupied flats, then the rent level increases. If, however, demand for housing is falling, then the rent level at this location decreases.

Two cases need to be distinguished:

- If $u(r)<\bar{u}$, then households are willing to move out of location $r$. It may be the case, however, that they cannot move if there is no supply of available housing at the other locations. In this case, there is no reason for rent levels 
to decrease. For this reason, decreasing rents are function of the proportion of unoccupied flats:

$$
\frac{d R_{H}}{d t}(r)=\frac{R_{H}(r)}{\tau_{R}} \cdot \phi\left(\frac{n(r) q(r)-H(r)}{n(r) q(r)}\right) \quad \text { if } u(r)<\bar{u}
$$

- If $u(r)>\bar{u}$, then households are willing to move in buildings, and rent level will increase in reaction:

$$
\frac{d R_{H}}{d t}(r)=\frac{R_{H}(r)}{\tau_{R}} \cdot \phi\left(\frac{n(r)+d(r)-\mu(r)}{n(r)}-\frac{H(r)}{n(r) q(r)}\right) \quad \text { if } u(r)>\bar{u}(\mathrm{~A}-18)
$$

In Eq. (A-18), the function $\phi$ is growing with respect to its argument, and has the same sign as its argument $(\phi(0)=0)$. Furthermore, we verify a posteriori in our numerical experiments that no housing service is provided beyond production capacity, that is: $n(r) q(r) \leq H(r)$ is always verified at all times and all locations.

\section{A.4 Specific functional forms}

In this section, we define the specific functional forms we will use in the rest of the article in order to explore the properties of our model.

\section{A.4.1 Basic functions}

Concerning the utility function, the transportation costs, the housing service production function, and the composite goods production function, we use functional forms that are considered as very classical in urban microeconomics ${ }^{7}$ : thus, the utility function, the housing service production function, and the general production function are Cobb-Douglas, while the transportation cost function is linear with respect to the distance from CBD.

$$
\begin{aligned}
& U(z, q)=z^{\alpha} q^{\beta} \text { where } \alpha, \beta>0 \text { and } \alpha+\beta=1 \\
& T(r)=p \cdot r \text { where } p>0 \\
& F(S, K)=A \cdot S^{a} \cdot K^{b} \text { where } a, b, A>0 \text { and } a+b=1 \\
& Y(N, K)=G \cdot N^{x} \cdot K^{y} \text { where } x, y, G>0 \text { and } x+y=1 \\
& \text { Land }(r)=l \cdot r \text { where } l>0
\end{aligned}
$$

$\overline{7}$ Those functional forms are widely used in urban economics for exploratory purpose, both because they allow advanced calculations, and because they reproduce realistic features of agents' preferences and of goods production. 
In this relationship, $p$ is the constant marginal transportation cost $^{8}$. The variable $G$ is the General Factor Productivity, while $A$ is the productivity associated to the production of housing service ${ }^{9}$.

\section{A.4.2 Dynamic evolutions}

Having defined the functional forms describing preferences, production, and transportation costs, we now turn to the dynamic relationships that need to be specified.

Housing consumption per capita: we specify here the expression of $\frac{\partial \tilde{u}}{\partial q}$, used in Eq. (A-8). With the functional forms we considered in Eq. (A-19), we have: $\frac{\delta \tilde{u}}{\tilde{u}}=\left(\frac{\beta}{q}-\frac{\alpha}{z} R_{H}\right) \delta q$. Furthermore, we choose the simplest specification for $g$, that is $g(x)=x$. Thus:

$$
\frac{\partial q(r)}{\partial t}=\left\{\begin{array}{l}
\frac{1}{\tau_{q}}\left(\frac{\beta}{q(r)}-\frac{\alpha}{z(r)} R_{H}(r)\right) \cdot q(r)^{2} \text { if } \psi(r)>0 \\
\frac{1}{\tau_{q}}\left(\frac{H(r)}{n(r)}-q(r)\right) \text { if } \psi(r) \leq 0
\end{array}\right.
$$

Moves: we specify the weight functions we use in Eqs. (A-10) and (A-11):

$$
w^{-}(u, \bar{u})=w^{+}(u, \bar{u})=\frac{2}{\pi} \arctan \left[w \cdot\left|\frac{u-\bar{u}}{\bar{u}}\right|\right]
$$

The function $\arctan (x)$ is increasing with respect to $x$, and converges towards $\pi / 2($ resp. $-\pi / 2)$ when $x$ goes towards $+\infty$ (resp. $-\infty)$, which ensures that $w^{+}$ and $w^{-}$have the desired properties. The coefficient $w$ in Eq. (A-21) modulates the strength of the force driving the moves.

Rent evolutions: in Eq. (A-18), we choose the simplest form for the function $\phi$, namely $\phi(x)=x$.

8 Thus, no congestion is taken into account, even though it is an important feature of transportation systems.

9 Concerning the static equilibrium and the calculations, see Appendix B 


\section{B Appendix: the static equilibrium of NEDUM}

\section{B.1 Static equilibrium: existence and unicity}

In this section, we show both existence and unicity of a static equilibrium defined by Eqs. (1) to (A-4).

Unicity: let us assume that such an equilibrium exists, and write all the variables values at this equilibrium with a superscript "**".

Concerning productive capital, Eq. (A-4) implies:

$$
I_{K}^{*}={\widetilde{I_{f K}}}^{*}=\delta_{K} \cdot K^{*}
$$

Meanwhile, from Eq.( A-3) we derive that:

$$
\delta_{K}+\rho^{*}=\frac{\partial Y}{\partial K}\left(N, K^{*}\right)
$$

Concerning housing capital, from Eq. (A-7), we get at any location $r$ :

$$
I_{f H}^{*}(r)=I_{H}^{*}(r)=\delta_{H} \cdot K_{H}^{*}(r)
$$

From Eq. (A-5), we also have:

$$
\delta_{H}+\rho^{*}=R_{H}^{*}(r) \frac{\partial F}{\partial K}\left(K_{H}^{*}(r), \operatorname{Land}(r)\right)
$$

The taking into account of the equilibrium utility level $u^{*}$, added to Eqs. (A-6) and (B-4), lead to a unique relationship:

$$
\widetilde{I_{H}}=j(\rho, K)
$$

where $j$ is decreasing in $\rho$ and increasing in $K$.

We now consider the system of four variables $\rho^{*}, I_{K}^{*}, \widetilde{I_{H}}{ }^{*}$ and $K^{*}$, and four equations (A-2), (B-1), (B-2), and (B-5).First, Eqs. (A-2) and (B-2) imply that ${\widetilde{I_{H}}}^{*}$ is increasing with respect to $K^{*}$. This relationship, added to Eq. (B-1), means that the derivative of LHS of Eq. (B-5) with respect to $K^{*}$ is greater 
than $\delta_{K}$. Meanwhile, we assume that the production function $Y(N, K)$ has decreasing marginal returns on capital, that go towards 0 as $K^{*}$ increases. As a conclusion, there is at most one possible equilibrium value for $K^{*}$.

Existence: if one assumes that, if $K^{*}=0$, the derivative of LHS of Eq. (B-5) with respect to $K^{*}$ is inferior to the derivative of $R H S$ of Eq. (B-5), then we also have the existence of the solution.

Since there is one and only one level of $K^{*}$ at equilibrium, then there is also one and only one level of corresponding consumption level $c$. At its stationary equilibrium, moreover, our model reproduces the features of classic urban microeconomics models (see for instance Fujita, 1989).

\section{B.2 Analytical calculations for the static equilibrium}

In this section, we characterize the static equilibrium with the functional forms defined by Eq. (A-19). We denote the equilibrium level of the variables with a superscript *. For instance Eqs. (A-5) and (B-3) give us for the equilibrium housing capital density at location $r$ :

$$
K_{H}^{*}(r)=\frac{b}{a} \frac{N p^{2}(\gamma+2)}{c^{*} \gamma+2} \frac{1}{\rho^{*}+\delta_{H}}\left(c^{*}-p \cdot r\right)^{\gamma+1} \cdot r
$$

This relationship, added to Eq. (A-6), implies that Eq. (B-5) translates into:

$$
\widetilde{I_{H}}=\frac{\delta_{H}}{\delta_{H}+\rho^{*}} \frac{b}{a} \frac{N c^{*}}{\gamma+3}
$$

Meanwhile, Eq. (B-2), which links the interest rate and the productive capital stock, becomes:

$$
\delta_{K}+\rho^{*}=y G\left(\frac{N}{K^{*}}\right)^{x}
$$

Using this relationship and Eq. (B-1), we derive:

$$
I_{K}^{*}=y \frac{\delta_{K}}{\rho^{*}+\delta_{K}}
$$

We can now consider Eqs. (A-2), (B-7) and (B-9), which imply that the equilibrium rate of interest is the unique solution of:

$$
s=\frac{\delta_{H}}{\rho^{*}+\delta_{H}} \frac{b}{a} \frac{1-s}{\gamma+2}+y \frac{\delta_{K}}{\rho^{*}+\delta_{K}}
$$

